\title{
Evaluación de programas públicos
}

José Adrián Chavarría Montenegro ${ }^{1}$

Economista Joseadricha55@gmail.com

Fecha recepción: abril 5 del 2016

Fecha aceptación: mayo 25 del 2016

Palabras claves: Programas públicos, evaluación de programas, lineamientos, cadena de valor público, indicadores de desempeño

Key words: Public programs, program evaluation, guidelines, public value chain, performance indicators

\section{ISSN $2308-782 X$}

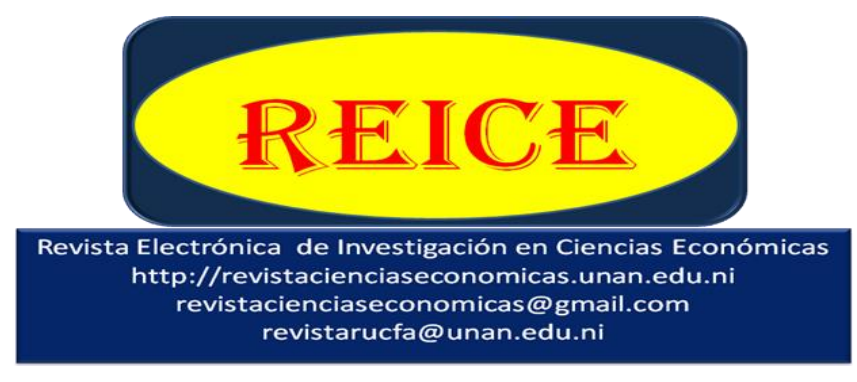

\section{Resumen}

El objetivo de este artículo es ahondar en los aspectos relevantes que se deben evaluar en un programa público presupuestado. Así como los aspectos conceptuales de la evaluación, tomando como referencia la aplicación del Marco Lógico como herramienta metodológica de evaluación de la estructura de los proyectos y de los programas públicos, a través de la cadena de valor público. El trabajo se fundamenta en la investigación de la literatura especializada, en el tema para el caso de los países de América Latina. Los resultados de la investigación

\footnotetext{
1 Artículo elaborado en el marco del desarrollo del programa de Doctorado en Ciencias Económicas en la Universidad del Zulia, Venezuela
}

indican que estos países han avanzado en el ámbito de la formulación, ejecución y evaluación de los planes y programas, sin embargo, en el ámbito de la evaluación los avances no son satisfactorios, específicamente en materia de seguimiento y evaluación de los programas públicos. Una de las razones que explica la situación anterior es que estos países, no disponen de una sólida base de datos que refleje los registros contables, financieros y patrimoniales de los programas y proyectos del sector público. Por tanto, un sólido sistema estadístico permitiría mejorar la información cualitativa y cuantitativa de calidad para calcular el costo de las metas de los programas y poder realizar de mejor forma el seguimiento y la evaluación física y financiera de los programas y proyectos del sector público

\section{Abstract}

The objective of this article is to explore more deeply in important aspects to be evaluated in a public budget program as well as conceptual aspects of evaluation in reference to the implementation of a logical framework as a methodological tool for assessing the structure of projects and public programs through a public value chain. The work is based on research of specialized literature on the subject in the case of Latin America. The research results indicate that these countries have made progress in the field of design, implementation and evaluation of plans and programs, however in the field of assessing progress it is not satisfactory, specifically in terms of monitoring and evaluation of public programs. One of the reasons that explain the above situation is that these countries do not have a solid database to reflect the accounting, financial and proprietary records of programs and projects in the public sector. Therefore, a strong statistical system would provide qualitative and quantitative quality information to calculate the cost of 
Evaluación de programas públicos

the program's goals in order to be able to

monitor the physical and financial evaluation of programs and projects in the public sector.

Introducción

REICE $\mid 106$

El siguiente artículo aborda aspectos relevantes que se deben tomar en consideración al momento de evaluar un programa público financiado vía presupuesto. En primer lugar, se destaca la importancia de evaluar la política pública y concretamente la de un programa público, éste a través de su ejecución y de sus resultados se concretizan los objetivos de una política pública.

También se destaca la evaluación del diseño de un programa, la gestión, los productos y usos de los mismos, considerados éstos como criterios fundamentales de la evaluación. Así mismo se destaca la metodología de evaluación a partir del análisis de los indicadores de producto, de resultado y de impactos de un programa a partir de la cadena de valor público, considerada ésta como un Marco Lógico de análisis y de evaluación de programas públicos.

En segundo lugar, se hace una breve descripción de los criterios de evaluación de los programas públicos en el país resaltando la importancia en tres aspectos fundamentales; primero, los programas públicos deben estar diseñados de tal manera que a través de ellos el gobierno alcance los objetivos estratégicos derivados de los lineamientos del Plan Nacional de Desarrollo Humano, por medio del marco presupuestario de mediano plazo y de los planes estratégicos institucionales.

También se aborda el tema de la calidad de la información contable y financiera que demanda la aplicación de un sistema de costeo de las metas establecidas en los programas públicos, este aspecto fortalece la información que la evaluación demanda.

Además, se abordan los sistemas de registros contables y financieros en los sistemas informáticos, los cuales permiten obtener información de calidad que 
permita la toma de decisiones respecto al estado de situación de un programa público.

\section{Material y métodos}

El tipo de Investigación que se lleva a cabo en la elaboración de este artículo, es explorativa, descriptiva y proyectiva, porque parte del análisis de los hechos en torno a la evaluación de programas públicos, que permiten llegar a reflexiones sobre cómo mejorar los procesos de evaluación de dichos programas.

La técnica de Investigación utilizada para la elaboración de este artículo, es principalmente la técnica de investigación documental y analítica, referida al tema de la evaluación de programas públicos.

El método de Investigación, permite conocer el objeto de la investigación no estudiado, por tanto, brinda información sobre la esencia del fenómeno observado, para establecer sus interrelaciones internas, pasando de la causa al efecto y de lo particular a lo general, a través del método inductivo de investigación. Este método se complementa con el análisis como método, dado que se analizan por separado los criterios a evaluar de un programa público, para llegar a conclusiones a través del método de Síntesis, y con ello tener una visión de conjunto del tema a investigar.

Resultado y discusión

Criterios para la evaluación de un programa público.

Para fortalecer el ciclo del presupuesto es necesario emprender acciones a fin de construir un sistema de evaluación del mismo, dado que en esta fase se conoce el grado de cumplimiento de las políticas, de las metas establecidas en los planes y programas estratégicos institucionales, así como la ejecución de los gastos e ingresos de las instituciones presupuestadas. 
Un programa público está enfocado a cumplir dos objetivos fundamentales: uno, que debe formularse en función de dar respuesta a una problemática en particular de una población meta, es decir, satisfacer las demandas de ese sector social y dos, la decisión política de los gobiernos de intervenir con el propósito de formular e implementar a través de las instituciones competentes, un conjunto de programas para cumplir con los objetivos que tienen los sectores públicos.

El programa se ubica en un sector o sub sector económico social y, su funcionamiento se desarrolla en el contexto económico, social, político, geográfico y cultural del país.

Un programa ubicado a nivel de una institución pública que atiende un sector en particular, se puede analizar y evaluar desde su estructura interna a través de un modelo descriptivo que expresa las principales estrategias de intervención por parte del gobierno ${ }^{2}$, porque sistematiza las medidas de políticas y la prestación de servicios, esto permite tener una mirada sistémica del accionar gubernamental sobre los problemas públicos (Sotelo, 2012).

El núcleo del modelo del programa lo constituye la cadena de valor público, expresada en Insumo-Producto-Resultado-Impacto, el cual ofrece un desarrollo que coloca esta cadena en un marco sistémico, a diferencia del enfoque de cadena de resultados, que renueva su significado al ponerlo en relación con componentes diversos y le otorga un marco interpretativo diferente al habitual. (Sotelo 2012).

Esta secuencia lógica también se conoce como cadena de resultado, que está presente en la propuesta de presupuesto por programa para el sector público

\footnotetext{
2Una estrategia de intervención pública es una combinación de medidas de política y de prestación de bienes y servicios; se pueden distinguir dos dimensiones de políticas, la de producción y de regulación.
} 
(Matus, Makon, Arrieche 1978) ${ }^{3}$ o en la planificación estratégica situacional (Matus 1978). ${ }^{4}$

Así mismo, ha sido presentada bajo el nombre de Marco Lógico, como herramienta para expresar la estructura de proyectos y generar indicadores de seguimiento y de evaluación del desempeño.

Esta última interpretación de la cadena de valor pública, como metodología del Marco Lógico, es la que se utiliza en general al momento de evaluar principalmente un programa público y analizarlo en toda su dimensión, tanto desde el punto de vista interno como externo.

La lógica de la cadena de valor es de carácter lineal, siendo de utilidad para la descripción, el análisis o la resolución de acciones que constituyen dimensiones particulares de una política pública.

En la cadena de valor el concepto de producto es relevante, porque en torno a él se establecen el resto de componentes de dicha cadena, porque toda política pública está orientada, en este caso, a la producción de bienes y servicios y a la parte normativa. La distinción entre medidas de política y prestación de bienes y servicios en la cadena de valor, resulta fundamental al momento de abordar la descripción y análisis de una política pública, en este caso la función de regulación es central en la producción de resultados e impactos que resulta inviabilizada en la cadena de valor.

Cuando se trata de evaluar los programas y los productos obtenidos, la experiencia indica que en los sectores público de América Latina existen instituciones, encargadas de formular y ejecutar programas que no tienen claridad sobre cuál es su producción terminal, es decir, ¿cuáles son los productos más

3Sotelo, 2012.

${ }^{4}$ Idem

REICE Vol. 4, No. 7, enero-junio 2016

ISSN: $2308-782 X$ 
importantes?, ¿cuáles son los usuarios más significativos?, ¿cuál es el gasto por producto?, y por tanto ¿cuál es su misión efectiva?; por lo general estas decisiones se dejan a la responsabilidad de las autoridades centrales, sin su correspondiente retroalimentación a nivel institucional.

Si el sector público produce bienes y servicios es porque existe la decisión política de modificar la realidad económica y social, a través de un programa público, situaciones que son objetos de políticas y de la intervención gubernamental, lo anterior plantea la necesidad de fortalecer los sistemas de evaluación.

Es decir, los sistemas de evaluación de los programas públicos se implementan y se conciben como una buena práctica, que permite mejorar la eficiencia y eficacia y sirvan de base para una mejor asignación de recursos ante la escasez de los mismos.

La evaluación del desempeño de un programa público se hace a través de un primer momento, que consiste en analizar la calidad del diseño enfocada al propósito de evaluar si el diseño de los programas está alineado a la producción final establecida en el marco de gasto institucional de mediano plazo y, si éste cumple con los lineamientos de políticas establecidas en los planes nacionales de gobierno.

Por tanto, los planes y los programas públicos deben de diseñarse en congruencia con los grandes lineamientos establecidos en los planes nacionales de gobierno $\mathrm{y}$, deben de evaluarse cualitativa y cuantitativamente en función de dichos lineamentos.

En un segundo momento se analiza la gestión del programa en el sentido de la eficiencia y la eficacia del mismo en términos de costos, recursos, tiempo y calidad de los productos brindados a la sociedad, además se debe evaluar la medición y 
el uso de los productos. Estos son los aspectos que se recomiendan cuando se trata de evaluar programas públicos.

De acuerdo a los criterios mencionados, existen dos escenarios que pueden darse en cuanto a la calidad de los programas públicos; en el sentido de que un programa público puede demostrar eficiencia en su ejecución física y financiera, pero a su vez puede ser ineficaz $y$, por el contrario, puede ser ineficiente pero eficaz respecto a los objetivos y finalidad del mismo. Por tanto, se debe procurar garantizar ambos criterios económicos para poder obtener mayor calidad en la gestión pública

La evaluación de los programas públicos se basa en información de calidad, para esto se necesita disponer de información cuantitativa y cualitativa objetiva, realista y fiable. A su vez la información que brinda el sistema de evaluación al desempeño es muy útil para la toma de decisiones a la hora de asignar recursos a un programa en vista a una nueva formulación presupuestaria.

Las instituciones públicas son los responsables de hacer las respectivas evaluaciones sobre el desempeño de los programas. Estas evaluaciones son muy útiles, si se hacen antes de la formulación de la política presupuestaria, de la asignación de los techos presupuestarios y de la formulación de los anteproyectos de presupuesto. De ésta manera se garantiza la eficiencia y la eficacia en la asignación de los recursos presupuestarios y, se mejora con ello, la calidad del ciclo presupuestario y por ende de la gestión pública.

En este caso, el sistema de seguimiento es un instrumento que contribuye a la gestión del sector público mediante un conjunto de indicadores que permiten verificar el cumplimiento de los objetivos gubernamentales, sectoriales y de sus expresiones cuantitativas. 
Revista Electrónica de Investigación en Ciencias Económicas

Facultad de Ciencias Económicas, UNAN-Managua

Evaluación de programas públicos

Es decir, el sistema de seguimiento o monitoreo se encarga de la recopilación sistemática de datos sobre indicadores de desempeño para proporcionar a los administradores y a los actores involucrados en las políticas, los programas y los proyectos, datos sobre el avance y el logro de los objetivos, así como sobre la utilización de los fondos asignados, en un lapso determinado (OCDE, 2002) ${ }^{5}$

El seguimiento busca conocer el avance en la consecución de los objetivos y de las metas del gobierno que, en la mayoría de los casos, están consignados en un Plan Nacional que se ejecuta con los recursos del presupuesto público .Por tanto, la función de seguimiento está estrechamente ligada a las de planificación y de presupuesto.

En cuanto a la evaluación, se convierte en una herramienta muy importante de gestión pública, porque permite conocer con mayor detalle del diseño y la gestión de un programa así como medir el uso de los productos, además, permite conocer las causas del buen o mal desempeño del mismo.

Por tanto, la evaluación es la "apreciación sistemática y objetiva de un proyecto, programa o política en curso o concluido, de su diseño, su puesta en práctica y sus resultados. El objetivo es determinar la pertinencia y el logro de los objetivos, así como la eficiencia, la eficacia, el impacto y la sostenibilidad para el desarrollo. Una evaluación deberá proporcionar información creíble y útil, que permita incorporar las enseñanzas aprendidas en el proceso de toma de decisiones" $(\mathrm{OCDE}, 2002)^{6}$.

La evaluación tanto desde el punto de vista de los programas público como de la gestión, se inserta en el marco teórico del análisis de las políticas públicas, en la

\footnotetext{
${ }^{5}$ Citado por Kaufmann, J., Sanginés,M., García, M., (2015). Construyendo gobiernos efectivos: Logros y retos de la gestión pública para resultados de América Latina y el Caribe. Washington.BID.

6 Idem.
}

REICE Vol. 4, No. 7, enero-junio 2016

ISSN: $2308-782 X$ 
medida que esta persigue producir información que tenga alguna relevancia con la toma de decisiones políticas-administrativas, información útil que permita resolver problemas concretos (Ballart, 1992) ${ }^{7}$

Otra definición, siempre enmarcada en el ámbito de los programas públicos es la que señala que la evaluación es la medición sistemática de la operación o impacto de un programa o política pública, comparada con estándares implícitos o explícitos en orden a contribuir a su mejoramiento (Weiss, 1998) ${ }^{8}$

La diferencia fundamental entre el seguimiento y la evaluación radica en que el primero ofrece información sobre la situación relativa al cumplimiento de los objetivos y efectos de una política, programa o proyecto. En cambio, la evaluación explica por qué esos objetivos o efectos se están logrando o no, y expone los cambios que se han producido en los beneficiarios y la sociedad. Mediante un proceso sistemático de recolección y de análisis de información relevante, la evaluación emite juicios sobre las causas y las razones de los resultados, examina resultados no buscados, estudia el proceso que se ha seguido para obtenerlos y proporciona recomendaciones para acciones futuras.

Es decir, la observación sistemática de la información relevante de un programa, a través del monitoreo y seguimiento, tienen como propósito introducir oportunamente ajustes o cambios necesarios que permitan reorientar las acciones para obtener los resultados esperados.

El programa define y formula un sistema de indicadores y establece metas de cumplimiento, el monitoreo, seguimiento y evaluación se hace en base a la medición cuantitativa y al análisis cualitativo de éstos indicadores y metas.

\footnotetext{
${ }^{7}$ Citado por Armijo,M., Planificación estratégica e indicadores de desempeño en el sector público, ILPES.

8 Idem.
} 
En este sentido, los componentes de la cadena de valor constituyen la base para la construcción de indicadores necesarios, indicadores de productos, de resultados y de impactos que permiten informar sobre los aspectos esenciales de la cadena de valor público de un programa.

A su vez, la interpretación analítica del conjunto de estos indicadores conforma la base de datos, que sirven de insumos para implementar la evaluación ${ }^{9}$ de los elementos de la cadena de valor de un programa público, empezando por las evaluaciones de impacto de resultados, de productos y de la cadena de valor en general, entendida esta como la viabilización de un programa y de una política pública.

Un aspecto crucial al momento de hacer la evaluación del programa, es la fiabilidad de la información, porque de ésta depende la calidad de la evaluación, en el sentido de que debe reflejar el verdadero estado de situación del programa. Por consiguiente, el uso de la evaluación es clave para que la autoridad tenga elementos de juicios y pueda tomar decisiones de hacer intervenciones o no, al programa, en el caso cuando se trate de una evaluación de un programa en marcha, para poder en concluir en el tiempo en que está previsto.

Cuando se habla de la información básica del programa que permite su evaluación, en cuanto a las metas e indicadores establecidos previamente, se refiere a si hay consistencia estadística de la información obtenida, si esta información se sustenta en una base de datos de registros que den indicios para la valoración y evaluación del programa como tal.

La consistencia estadística contable, financiera y presupuestaria es útil, entre otros aspectos, para diagnosticar la situación del sector público en el contexto

\footnotetext{
${ }^{9}$ Ya que esta se atenga a lo planificado o busque efectos con prescindencia de los objetivos establecidos.
} 
socioeconómico del país, definir los objetivos y metas nacionales, efectuar un seguimiento de estos últimos, y analizar los elementos que afectan el desempeño de las políticas, los programas y los proyectos gubernamentales.

El diseño del programa debe enfocarse a satisfacer una demanda de la población que no ha sido satisfecho por diversas razones. Para que el programa pueda concretizarse y tenga sus resultados e impactos respectivos, debe incluirse e la formulación presupuestaria que hace la institución encargada del programa.

Este proceso debe de garantizar la efectiva coordinación entre las instancias de planificación y de presupuesto a lo interno de la institución, en el sentido de que se debe garantizar que la estructura programática del presupuesto permita incorporar el programa al presupuesto de mediano plazo con su respectiva asignación presupuestaria.

La ejecución del programa permitiría, de esta manera, que la estructura programática refleje, en su dimensión los indicadores y metas que el programa tiene diseñado.

Al respecto la experiencia en los países de América Latina indica que el logro de esta coordinación no siempre se alcanza, cuando se plantean las siguientes interrogantes: ¿Que financia el presupuesto?, ¿el presupuesto a que plan corresponde?, estas interrogantes no siempre han tenido un criterio único, al contrario, hay una diversidad de opiniones relacionado al tema.

En este sentido (Martirene, 2007), plantea que hay países que planifican sin presupuestar y países que presupuestan sin planificar, reflejando en sentido extremo con esta frase, la poca coordinación de las instancias encargadas de estos procesos.

La experiencia al respecto indica, en este caso, que las oficinas encargadas de formular el presupuesto, si bien es cierto, reconocen la existencia de ciertas 
prioridades en los planes, programas y proyectos, no necesariamente en el momento de formular el presupuesto lo toman en cuenta, por su lado las oficinas de planificación reconocen que los presupuestos se formulan siguiendo otros criterios y no necesariamente los planes, (Shack 2008).

En el país se ha avanzado en la formulación y ejecución presupuestaria de acuerdo con lo establecido en el artículo 65 y 66 de la Ley de Administración Financiera y del Régimen Presupuestario.

La Ley, delega a las instancias pertinentes, la responsabilidad de evaluar el cumplimiento de las metas, de los planes y programas, a fin de exponer su opinión técnica respecto a la eficiencia operacional de los organismos y entidades, teniendo en cuenta la ejecución física, financiera y económica.

El artículo 65 establece que debe de realizarse "en forma trimestral y al cierre presupuestario, un análisis de los resultados financieros y físicos y, una evaluación de los programas de gastos y de cualquier otra información que se considere pertinente. Con éste análisis se deben evaluar los efectos producidos de la gestión presupuestaria, interpretando las variaciones operadas con respecto a lo programado e identificar las causas.

Por su parte, en virtud del artículo 66, se deben preparar informes con recomendaciones a las autoridades superiores y a los responsables de los organismos y entidades que correspondan.

Esta información se refiere a los informes de ejecución presupuestaria de las instituciones del Gobierno Central, los cuales deben de remitirse a la Contraloría General de la República y a la Asamblea Nacional, donde se informa el grado de ejecución física y financiera del gasto público de acuerdo a sus fuentes de financiamiento y su clasificación en gasto corriente y de capital, así como el monto de los ingresos públicos percibidos. 
La formulación de los programas presupuestarios es una vía para lograr que el presupuesto se formule en base a políticas públicas y no en incrementos automáticos en función de los recursos previstos.

En este caso, el programa es la estrategia a través de la que se logran los objetivos del plan de Gobierno, mediante la gestión de los programas y proyectos, las identidades gubernamentales concretizan la producción de bienes y servicios para los ciudadanos y por tanto la creación de valor público.

En concordancia con los Planes Nacionales, los Marcos de Gastos Institucionales de Mediano Plazo y los Presupuestos anuales también de Mediano Plazo y orientados a resultados, el programa presupuestario expresa la estrategia y la política de la institución.

En términos generales, un programa institucional debe formularse de acuerdo a la estrategia del sector al que pertenece $y$, a su vez debe ser consistente con los lineamientos de Plan Nacional de Desarrollo Humano respecto a dicho sector.

Lo anterior debe ser congruente con la formulación de un programa al momento de formular indicadores, metas y compromisos de Buen Gobierno. Recordando el objetivo de todo sector público, que consiste en llegar a la sociedad ofertando bienes y servicios públicos, en este caso, la estrategia del plan y los programas deben estar diseñados en función de suplir una demanda de la sociedad.

De esta manera, el presupuesto de mediano plazo ${ }^{10}$ enfocado a resultados, busca mejorar la coherencia entre las políticas nacionales y las proyecciones de egresos, así como el control y la medición de los resultados, de los diferentes programas nacionales priorizados en los presupuestos estatales, para ello define objetivos, metas y se asigna el presupuesto que se estime adecuado. Para comprometer

\footnotetext{
10 Es la técnica presupuestaria que utiliza el gobierno para proyectar las variables fiscales a mediano plazo como una herramienta de gestión pública.
}

REICE Vol. 4, No. 7, enero-junio 2016

ISSN: $2308-782 X$ 
ciertos resultados de los programas, las instituciones deben de realizar un proceso de planificación estratégica.

El presupuesto por resultado es una propuesta de gestión gerencial que trata de encontrar el método más eficiente de lograr un objetivo determinado y, al mismo tiempo la eficacia de las instituciones.

El hecho de que las instituciones formulen presupuestos en base a programas, permiten profundizar en su estructura y, en las relaciones pertinentes en la cadena de resultados: insumos-productos-resultados-impacto. De esta manera, el programa está orientado a contribuir a la consecución de los objetivos de la política de una institución o de un sector. Lo anterior demuestra la importancia del programa presupuestario, en la medida que se convierte en un centro de producción y de asignación de recursos institucionales.

Es por ello que el programa presupuestario debe estar orientado a la consecución de determinada política, la cual debe estar en consonancia con los lineamientos, estrategias de mediano y largo plazo establecidas en el Plan Nacional de

Desarrollo Humano.

La estructura programática institucional del presupuesto anual es por categoría u objeto del gasto, derivándose en programas, sub programas, proyectos, actividades y obras. Esta estructura permite mayor flexibilidad y facilita a las instituciones los traslados presupuestarios entre programas, subprogramas y proyectos.

El presupuesto por programa es la base para la implementación de un presupuesto por resultados, el cual debe ir acompañado de información, acerca del desempeño de los resultados de la gestión presupuestaria. 
Para alcanzar este objetivo, es necesario el desarrollo de un sistema de información sobre el desempeño de las finanzas públicas, a través de la construcción de indicadores de desempeño y de un sistema de evaluación de los programas públicos. Es decir, contar con un sistema de información que facilite al Gobierno el monitoreo y la evaluación de sus programas, constatar sí efectivamente, se están alcanzando los objetivos y los resultados esperados.

El presupuesto de mediano plazo, en el marco de una gestión orientada a resultados, debe expresar las responsabilidades asignadas a las instituciones, públicas en función de las políticas, objetivos y metas contempladas en los planes estratégicos nacionales, sectoriales e institucionales.

Además, un método eficaz para medir la eficiencia y eficacia de un programa de Gobierno implementado por una institución, precisamente es a través del sistema de indicadores mencionados, que son una unidad de medida que permite el seguimiento y evaluación periódica de las variables claves de un programa o proyecto.

La correcta definición de los indicadores es fundamental para poder evaluar el desempeño de las instituciones en el cumplimiento de sus metas, por tanto los indicadores deben de tener las siguientes características: medibles y comparables, en términos de calidad, costo y tipo de beneficiario; específicos, dirigidos a un área de competencia claramente definida; accesibles, económicos y confiables, la información que sirva de base para el cálculo de los indicadores, debe poder ser recolectada fácilmente, a bajo costo y relevantes, deben referirse a productos 0 resultados de la institución; simples, comprensibles y precisos, deben de procurarse que los indicadores seleccionados puedan ser entendidos por la población en general; públicos y transparentes, los indicadores deben ser conocidos y accesibles a todos los funcionarios que corresponda. En síntesis, los indicadores deben de reflejar la misión del sector público y de la institución en particular. 
La evaluación física de las obras es muy importante porque abarca los productos de un proyecto, es decir, la cobertura total del proyecto, desde su formulación hasta su seguimiento y evaluación, sin embargo, para que esta evaluación se cumpla satisfactoriamente se hace uso del sistema de indicadores y seguimiento de los resultados.

Por tanto, se hace necesario fortalecer el sistema de seguimiento físico que permita alinear a mediano plazo las políticas sectoriales a las proyecciones institucionales de gasto, al mismo tiempo que garantice el monitoreo y la evaluación de la ejecución concretamente en la inversión en obras públicas.

Para el caso de la evaluación de los programas presupuestarios, se consideran apropiados los siguientes criterios.

Calidad del diseño del programa, bajo este criterio se propone evaluar la consistencia del diseño actual del programa presupuestario en base a tres aspectos; Consistencia en el tiempo, coherencia con lo planificado durante el periodo de evaluación; consistencia interna, consistencia entre la lógica del programa y su cadena de producción; consistencia con la planificación nacional, contribución de los programas a las prioridades que el Gobierno establece en el corto y largo plazo.

Esto significa que todos los programas de inversión pública estén a lineados al Plan Nacional de Desarrollo Humano, a los Planes de Buen Gobierno y al Programa Estratégico Institucional.

Los programas están clasificados por actividades en el presupuesto, es decir, los programas se diseñan de acuerdo a la técnica presupuestaria implementada para analizar las respectivas asignaciones presupuestarias, de esta forma se facilitaría hacer el seguimiento y la evaluación financiera. 
Los programas están enfocados a obtener resultados medibles, en base a la construcción de indicadores y la definición de metas cualitativas y cuantitativas, que facilita el alcance y el cumplimiento de las metas establecidas en cada programa.

La Gestión del programa, es la eficiencia en la generación de productos previstos, con ello se pretende evaluar si la institución está obteniendo los productos finales, según lo planificado y, posteriormente analizar las causas de las desviaciones, en el caso de que hubiera. Con ello se persigue documentar y analizar las buenas prácticas de gestión o, sugerir una mejor adecuación entre insumos y productos.

Para ello es preciso que la financiación del programa debe estar garantizada en el presupuesto anual y de mediano plazo, esto con el propósito de evitar el surgimiento de brechas financieras, la estructura institucional debe ser adecuada al perfil del programa y a su vez debe de garantizar la coordinación intra e interinstitucional.

La medición y el uso de los productos, es un criterio que valora hasta qué punto existen capacidades instaladas, para una correcta medición del uso de los productos, bienes y servicios, provistos por el programa presupuestario, además se evalúa el uso de los productos generados en términos de cobertura geográfica y de población meta.

Respecto a la evaluación presupuestaria, se dispone de un sistema de información sobre el desempeño de las finanzas públicas a través de la construcción de indicadores de desempeño y de un sistema de evaluación de los programas públicos. Es decir, se dispone de un sistema de información que facilite al Gobierno el monitoreo y la evaluación de sus programas, constatar sí efectivamente se están alcanzando los objetivos y los resultados esperados.

Al respecto, hay un flujo de información que proviene de los diferentes sistemas informáticos empleados, para registrar programación y ejecución presupuestaria 
tanto del gasto corriente como del gasto de capital y, también hay información proveniente del sistema informático del Gobierno.

Cada uno aporta información relevante del avance de ejecución física y financiera del gasto público, concretizado en los programas y proyectos públicos que se registran en el sistema de información presupuestaria, avance de las metas establecidas, en cada plan estratégico institucional.

La información sobre la ejecución física y financiera de los programas de inversión pública se registra en base de los avalúos que son entregados por los contratistas a las instituciones. También se registra la ejecución presupuestaria del gasto corriente.

Los sistemas reportan información de las metas de los programas y proyectos reportados al gobierno y, las metas reportadas en los sistemas de información de gestión financiera.

Por lo general los sistemas tienen niveles de agregación y desagregación diferentes. Los vínculos de estos flujos de información, permite obtener en tiempo real la ejecución física y financiera del gasto presupuestario, que previamente se han asignado, para cumplir con las metas y los objetivos de política establecidos en el plan estratégico institucional.

El vínculo de las metas y el presupuesto a nivel institucional se extiende al vínculo entre los distintos sistemas de información, lo que permite realizar con mayor eficiencia el seguimiento y la evaluación de la ejecución presupuestaria y, del grado de cumplimiento de las metas que el gobierno se plantea, para alcanzar los objetivos de la política pública.

Por tanto, el proceso de seguimiento físico y financiero de las metas institucionales y del presupuesto se dan por medio de la articulación o vínculo 
informático: Entre los sistema de información sobre los planes, metas e indicadores, que utiliza el Sistema de Integración del Gobierno de Reconciliación y Unidad Nacional; entre la ejecución física y financiera del programa de inversión pública que utiliza el Banco de Proyectos del Sistema Nacional de Inversión Pública; y sobre la ejecución física y financiera del presupuesto que utiliza el Sistema Integrado de Gestión Financiera y Auditoria.

Estos sistemas, generan un flujo de información en tiempo real de la ejecución física y financiera de los recursos presupuestarios asignados a metas institucionales de un programa.

Un aspecto muy importante para fortalecer el sistema de evaluación de los programas públicos, es el costo asociado a la asignación de recursos para el logro de una meta, particularmente cuando se trata de la producción vinculada a un servicio social asociado a una meta. Aquí la mayoría de los países latinoamericanos presentan dificultades en el costeo de las metas de los programas institucionales.

Al respecto, la institución por medio del presupuesto asigna recursos para alcanzar la meta de un programa, sin embargo, esto no implica tener información disponible acerca de lo que cuesta dicha meta. Para el logro, de lo anterior se debe de fortalecer el sistema de costeo analítico ${ }^{11}$ de los servicios que se producen y se ofertan a la sociedad.

Porque el sistema de costeo, permite clasificar los insumos, en costos fijos y costos variables, necesarios para producir un bien o servicio público y, por tanto, conocer el costo unitario de la producción de un bien o un servicio.

\footnotetext{
11 Son muy pocos los países en América Latina que han implementado un sistema de costeo de los bienes y servicios producidos por un programa público.
}

REICE Vol. 4, No. 7, enero-junio 2016

ISSN: $2308-782 X$ 
Los responsables de las áreas de finanzas, de contabilidad y de recursos humanos, en coordinación con las áreas de planificación ${ }^{12}$, realizan acciones tendientes a obtener una aproximación del sistema de costeo de los servicios públicos producidos y, con ello el costo de las metas.

Por tanto, se debe fortalecer el sistema de costeo a través de las siguientes acciones: Clasificar los servicios que están vinculados al cumplimiento de metas de un programa, así como cuantificar los recursos humanos, materiales y físicos, para cada uno de los servicios vinculados con las metas $y$, calcular los costos unitarios para los servicios, vinculados al cumplimento de metas, así como determinar costo de la meta, a partir de los costos unitarios de los insumos asociados a dicha meta.

La institución a partir de la formulación de su plan operativo, anual define las metas establecidas en su plan estratégico institucional, esas metas deben ir vinculada a los servicios que presta dicha institución. Por tanto, la institución precisa los bienes y servicios que obtiene vinculados a una meta en particular.

Además, procura los insumos necesarios para la producción del bien o el servicio, asignándoles sus respectivos valores a través del cálculo de los costos variables y costos fijos. Posteriormente se establece el cálculo del costo unitario por servicios. En términos generales, para garantizar una mejor evaluación de los programas públicos, se deben actualizar o formular metodologías de evaluación con el propósito de brindar información de calidad para garantizar una mejor asignación de recursos presupuestarios.

\footnotetext{
12 Estas son las áreas de las instituciones públicas que disponen de información de costo y bienes y servicios producidos
}

REICE Vol. 4, No. 7, enero-junio 2016

ISSN: $2308-782 X$ 


\section{Revista Electrónica de Investigación en Ciencias Económicas \\ Facultad de Ciencias Económicas, UNAN-Managua \\ Evaluación de programas públicos}

Conclusiones

los criterios de evaluación de los programas públicos están referidos a evaluar la calidad del diseño, la gestión, medición y uso de los productos y servicios obtenidos de dichos programas.

La construcción y evaluación de un sistema de indicadores, que conforman un programa, de productos, de resultados y de impactos, brindan información necesaria para medir la eficiencia, la eficacia y la calidad del gasto público asignado a los programas.

Además la evaluación permite mejorar la asignación de los recursos presupuestarios, dado que las autoridades a partir de dicha evaluación y considerando las prioridades de las políticas públicas, están en mejores condiciones para evaluar el desempeño de los programas públicos.

Por tanto, la evaluación de los programas públicos es una herramienta de planificación, a su vez, muy útil para mejorar la calidad de la gestión pública, dado que a través de sus resultados se obtiene una información cualitativa y cuantitativa de calidad, la cual sirve para insumo a las autoridades para la toma de decisiones presupuestarias y de políticas públicas.

Bibliografía.

Armijo, M., Planificación estratégica e indicadores de desempeño en el sector público, ILPES.

Bueno, C Y Osuna, L. (octubre, 2013). Evaluación del diseño de políticas públicas: propuesta de un modelo integral. Revista del CLAD, Reforma y Democracia N57. Caracas. 
González, Y. (septiembre 2011). Planificación Sectorial, Territorial e Institucional, Apoyo a la instrumentación de un sistema de gestión por resultados. PRODEV.

Kaufmann, J., Sanginés, M., García, M., (2015). Construyendo gobiernos efectivos: Logros y retos de la gestión pública para resultados de America Latina y el Caribe. Washington. BID.

Ley 290: Ley de Administración Financiera y del Régimen Presupuestario de Nicaragua 2005.

Martirene, R. (junio, 2007). Modelo de integración de un Plan Estratégico Institucional al Presupuesto Público orientado a Resultados. XXI Seminario Nacional de Presupuesto Público. Olavarría, M. (octubre 2012). La evaluación de programas en Chile: Análisis de una muestra de programas públicos. Revista CLAD, Reforma y Democracia N54. Caracas.

Schack, N. (junio, 2008). Introducción caracterizar la articulación entre el plan $y$ el presupuesto. Algunas experiencias en América Latina. Documento presentado en la IV reunión en el Desarrollo y Gestión para el resultado realizado por el BID/PRODEV. Ciudad de México.

Sotelo M y Aníbal, J. (2012). La Cadena de valor público: Un principio ordenador que previene la colisión metodológica. ASIP. 